\title{
CIENCIAMATRIA
}

Revista Interdisciplinaria de Humanidades, Educación, Ciencia y Tecnología

Año VII. Vol. VII. N¹2. Enero - Junio. 2021

Hecho el depósito de ley: pp201602FA4721

ISSN-L: 2542-3029; ISSN: 2610-802X

Universidad Nacional Experimental Francisco de Miranda (UNEFM). Santa Ana de Coro. Venezuela

Karen Lisseth Apolo-Pérez; Juan Edmundo Álvarez-Gavilanes;

William Giovanni Vicuña-Matute

$\underline{\text { DOI } 10.35381 / \mathrm{cm} . v 7 i 12.419}$

\section{Gestión estratégica del talento humano en el Hospital General Machala}

Strategic management of human talent for job skills at the General Machala Hospital

\author{
Karen Lisseth Apolo-Pérez \\ karen.apolo.78@est.ucacue.edu.ec \\ Universidad Católica de Cuenca, Cuenca \\ Ecuador \\ https://orcid.org/0000-0002-9485-8221 \\ Juan Edmundo Álvarez-Gavilanes \\ juan.alvarezg@ucacue.edu.ec \\ Universidad Católica de Cuenca, Cuenca \\ Ecuador \\ https://orcid.org/0000-0003-0978-3235 \\ William Giovanni Vicuña-Matute \\ wgvicunam@ucacue.edu.ec \\ Universidad Católica de Cuenca, Cuenca \\ Ecuador \\ https://orcid.org/0000-0001-7041-5867
}

Recibido: 01 de octubre de 2020

Aprobado: 15 de diciembre de 2020 


\author{
CIENCIAMATRIA \\ Revista Interdisciplinaria de Humanidades, Educación, Ciencia y Tecnología \\ Año VII. Vol. VII. N¹2. Enero - Junio. 2021 \\ Hecho el depósito de ley: pp201602FA4721 \\ ISSN-L: 2542-3029; ISSN: 2610-802X \\ Universidad Nacional Experimental Francisco de Miranda (UNEFM). Santa Ana de Coro. Venezuela \\ Karen Lisseth Apolo-Pérez; Juan Edmundo Álvarez-Gavilanes; \\ William Giovanni Vicuña-Matute
}

\title{
RESUMEN
}

En el mundo de la gestión y desarrollo del talento humano, la capacitación continua es una pieza fundamental. El objetivo de esta investigación es mejorar el modelo de gestión estratégica del HGM y el apoyo a su formación del personal. La investigación fue de tipo descriptiva - trasversal. Se encontró que no existe un adecuado proceso de inducción y capacitación al talento humano, al igual que la inexistente evaluación y control que genera un desempeño laboral inadecuado. El modelo de gestión estratégica aporta sustancialmente al mejoramiento de los procesos, así como también al logro de los objetivos institucionales que busca el mejoramiento del servicio permanentemente.

Descriptores: Hospital; gestión de recursos; gestión del personal. (Palabras tomadas del Tesauro UNESCO).

\begin{abstract}
In the world of human talent management and development, continuous training is a fundamental piece. The objective of this research is to improve the strategic management model of the HGM and to support its staff training. The research was descriptive transversal. It was found that there is no adequate induction and training process for human talent, as well as the non-existent evaluation and control that generates an inadequate job performance. The strategic management model contributes substantially to the improvement of the processes, as well as to the achievement of the institutional objectives that the improvement of the service seeks permanently.
\end{abstract}

Descriptors: Hospitals; resources management; personnel management. (Words taken from the UNESCO Thesaurus). 


\section{CIENCIAMATRIA}

Revista Interdisciplinaria de Humanidades, Educación, Ciencia y Tecnología

Año VII. Vol. VII. N¹2. Enero - Junio. 2021

Hecho el depósito de ley: pp201602FA4721

ISSN-L: 2542-3029; ISSN: 2610-802X

Universidad Nacional Experimental Francisco de Miranda (UNEFM). Santa Ana de Coro. Venezuela

Karen Lisseth Apolo-Pérez; Juan Edmundo Álvarez-Gavilanes;

William Giovanni Vicuña-Matute

\section{INTRODUCCIÓN}

La gestión estratégica es la técnica de conocer, implementar y evaluar las decisiones actuales o futuras que permite a una institución, realizar eficientemente la distribución de los recursos para lograr los objetivos planteados. Uno de los principales recursos con que cuenta cada institución es el talento humano, siendo una parte clave que gestiona el desempeño de sus trabajadores acorde con las capacidades y actitudes de cada persona para alcanzar la mejor solución de sus procesos (Mendoza-Novillo, et al., 2019).

Es importante el reconocimiento de las personas dentro de las instituciones ya que motiva gran interés, permitiendo a la institución contar con talento humano capacitado y apto para ocupar estratégicamente los puestos de gestión asignados que fomenten a la productividad de la institución (López-Puig, et al., 2017). Uno de los principios fundamentales de la gestión del talento humano es elaborar un esquema de cualidades competitivas que generen un escenario donde se puedan aplicar políticas para un mejoramiento de la estructura organizacional favorable para la institución que permitan la toma de decisiones gerenciales que garanticen la continuidad de los procesos proyectándolos para el futuro de la empresa.

La idea es contar con el personal idóneo, siendo el sector de la salud más preponderante del manejo eficiente de este recurso humano, señalando que también la calidad de atención es un factor importante que debe tomarse en cuenta al momento de brindar un servicio de salud. La gestión estratégica en los servicios de salud, ayuda a diseñar planes a mediano y largo plazo, permitiendo establecer objetivos claros y definidos con proyección a realizar acciones encaminadas en el logro de los resultados esperados de la institución, además nos permite contar con profesionales capacitados para salvaguardar el buen funcionamiento de un servicio específico, logrando optimizar los tiempos y recursos para la unidad médica (Bernal-González, et al., 2014).

El Ecuador cuenta con un total de 3.446.724 personas afiliadas a la Seguridad Social "IESS", quienes entre sus beneficios poseen el derecho a la atención integral en salud.

El Instituto Ecuatoriano de Seguridad Social en la ciudad de Machala cuenta con un 


\section{CIENCIAMATRIA}

Revista Interdisciplinaria de Humanidades, Educación, Ciencia y Tecnología

Año VII. Vol. VII. N¹2. Enero - Junio. 2021

Hecho el depósito de ley: pp201602FA4721

ISSN-L: 2542-3029; ISSN: 2610-802X

Universidad Nacional Experimental Francisco de Miranda (UNEFM). Santa Ana de Coro. Venezuela

Karen Lisseth Apolo-Pérez; Juan Edmundo Álvarez-Gavilanes;

William Giovanni Vicuña-Matute

hospital de referencia en la provincia de El Oro que brindan comodidad y tecnología de punta, dando un giro trascendental en la calidad de atención a los afiliados, mediante Resolución No. C.D. 546, de fecha 2 de marzo de 2017 se aprobó la reestructuración del Hospital General Machala (HGM), se aprobaron 378 puestos entre profesionales de la salud, apoyo de la salud y administrativos para las diferentes áreas de este nosocomio (Instituto Ecuatoriano de Seguridad Social, 2017).

De la muestra tomada en el HGM se puede considerar que una gran parte del personal que labora en la institución no ha recibido capacitaciones que le permitan desempeñarse de forma eficiente en su puesto de trabajo, provocando una desmotivación generalizada y un manejo poco eficiente del talento humano, todo lo mencionado anteriormente nos da como consecuencia que la institución realice procesos repetitivos, informes ineficientes que demandan de revisiones periódicas, ocupando tiempo innecesario que retrasa la gestión hospitalaria, la misma que ocasiona un impacto negativo en la atención directa de los afiliados.

\section{Referencial Teórico}

\section{El talento humano y la gestión para una administración eficiente}

Se define al talento humano como una serie de conocimientos, experiencias acumuladas, capacidades intelectuales, técnicas, destrezas que posee cada individuo y que aporta a la institución, provocando que se convierta en una empresa competitiva, garantizando que las diferentes áreas funcionen de forma óptima aprovechando al máximo sus recursos (Armijos-Mayon, et al., 2019). Se considera al talento humano como la herramienta más importante de la organización y de ella dependen el éxito o fracaso de las mismas, la concepción de este recurso debe ser desde el punto de vista de inversión y no como un costo, siendo ser la prioridad en toda institución.

Para quienes han realizado investigaciones relacionadas con la gestión del talento humano, la definen al conjunto de acciones que realizan las personas usando sus capacidades y competencias en servicios o áreas específicas, apoyado el crecimiento 


\section{CIENCIAMATRIA}

Revista Interdisciplinaria de Humanidades, Educación, Ciencia y Tecnología

Año VII. Vol. VII. N¹2. Enero - Junio. 2021

Hecho el depósito de ley: pp201602FA4721

ISSN-L: 2542-3029; ISSN: 2610-802X

Universidad Nacional Experimental Francisco de Miranda (UNEFM). Santa Ana de Coro. Venezuela

Karen Lisseth Apolo-Pérez; Juan Edmundo Álvarez-Gavilanes;

William Giovanni Vicuña-Matute

organizacional, estableciendo mejoras las condiciones laborales dentro de su entorno organizativo (Ramírez, et al., 2019).

Para una administración eficiente es fundamental contar con una correcta planificación, organización, coordinación y optimización de forma eficiente del personal de una institución, por lo que es necesario la utilización de herramientas y técnicas que mejoren el ambiente laboral de cada departamento optimizando la comunicación interna entre compañeros, permitiendo lograr objetivos personales que beneficien directamente el trabajo que desarrollen (Armijos-Mayon, et al., 2019). Como se indica en el párrafo anterior podemos entender que la gestión de la administración del talento humano es la acción de gestionar: lo que se entendería dentro del contexto empresarial cómo aplicar acciones que permitan lograr objetivos que beneficien a la institución, empresa u organización.

La gestión eficiente del talento humano permite establecer un clima laboral óptimo para lo cual es necesario de la permanencia del personal evitando la rotación exagerada del mismo y la actitud que manejen en sus labores cotidianas (Vera-Barbosa \& Blanco-Ariza, 2019). La gestión del talento humano, no solo debe enfocarse hacia el usuario interno de la organización si no tomarlo como un conjunto que interactúa entre si donde existe una integración efectiva de sus usuarios externos, donde todos juegan un rol importante para el desarrollo exitoso.

La organización debe asignar cada puesto de trabajo al personal idóneo, con perfiles acordes a lo que requiere la empresa, quienes debe cumplir con los requerimientos establecidos dentro de la institución. En otras palabras, que cada cargo deba estar ocupado con los requerimientos mínimos acorde a las funciones que el puesto exige, por lo que el diseño y aplicación de un manual de puestos es imperativo ya que sería una herramienta que facilite el trabajo de reclutamiento y selección de personal idóneo para la empresa (Mendivel-Gerónimo, et al., 2020).

La gestión del talento humano se enfoca en la incorporación de nuevo personal que hagan de la empresa su prioridad dándole el valor agregado que necesita (Gonzáles- 


\section{CIENCIAMATRIA \\ Revista Interdisciplinaria de Humanidades, Educación, Ciencia y Tecnología \\ Año VII. Vol. VII. N¹2. Enero - Junio. 2021 \\ Hecho el depósito de ley: pp201602FA4721 \\ ISSN-L: 2542-3029; ISSN: 2610-802X \\ Universidad Nacional Experimental Francisco de Miranda (UNEFM). Santa Ana de Coro. Venezuela}

Karen Lisseth Apolo-Pérez; Juan Edmundo Álvarez-Gavilanes;

William Giovanni Vicuña-Matute

Ramos \& Molina-Gómez, 2016). Por lo tanto, es indispensable que exista un efectivo compromiso entre la alta gerencia y la gestión del talento humano, siendo la base fundamental para el éxito de todo tipo de sistema de gestión de calidad (Romero-Parra \& Salcedo-Lara, 2019).

Parte primordial de los sistemas de gestión del talento humano es precisar que asegure su funcionamiento normal y desempeño para la eficacia del sistema. De ahí la importancia del fortalecimiento de las competencias (conciencia, conocimiento, liderazgo y compromiso) pilares importantes para la aplicación de los principios de calidad dirigidos específicamente en la atención del usuario. El principal activo que existe en toda organización o empresa es el talento humano y por tal motivo debe ser tratado de forma delicada, ya que de este factor depende el éxito o fracaso de la misma, cabe indicar que el personal debe contar con una buena comunicación, destrezas, actitudes, trabajo efectivo en equipo dejando de lado temas personales que pueden interferir y que son esenciales para el buen funcionamiento de un área o servicio (Mendivel-Gerónimo, et al., 2020).

Es sustancial que cada empresa o institución cuente con un área encargada de cuidar el bienestar y promover efectivamente el desarrollo profesional de cada trabajador desde sus inicios a fin, las cuales son: la selección efectiva, evaluación psicológica, la capacitación y el desarrollo intelectual, y el clima laboral en el que se desenvuelven (Chávez-Epiquén, et al., 2018). Podemos definir que el éxito de la gestión dentro de una organización depende directamente de la gestión estratégica por cuanto es una actividad de importancia relevante que debe ir de la mano con los objetivos establecidos (AscencioVargas, et al., 2016).

En el mundo de la gestión y desarrollo del talento humano, la capacitación continua es una pieza fundamental que interviene en el nivel de respuesta que un empleado pueda dar ante los requerimientos que la empresa realice, a esto se conoce como actividades organizadas y orientadas a lograr un cambio que fomenta el conocimiento del personal explotando las destrezas y virtudes en la formación del talento humano de la empresa 


\section{CIENCIAMATRIA}

Revista Interdisciplinaria de Humanidades, Educación, Ciencia y Tecnología

Año VII. Vol. VII. N¹2. Enero - Junio. 2021

Hecho el depósito de ley: pp201602FA4721

ISSN-L: 2542-3029; ISSN: 2610-802X

Universidad Nacional Experimental Francisco de Miranda (UNEFM). Santa Ana de Coro. Venezuela

Karen Lisseth Apolo-Pérez; Juan Edmundo Álvarez-Gavilanes;

William Giovanni Vicuña-Matute

(Soto-Berenguel, 2003).

El análisis de las necesidades de formación del personal es un punto clave para asegurar el éxito, indicando que la capacitación continua se transforma dentro de la organización en una herramienta importante que permite mejorar e incrementar los conocimientos de sus empleados convirtiéndose en un activo invalorable. Se puede definir que conocer las falencias del conocimiento del personal permite a la empresa establecer las prioridades dentro del periodo de formación de la organización. Organizar las eventualidades que pueden ocurrir frente a los resultados obtenidos del aprendizaje y el impacto que tendrá frente al desarrollo de los trabajadores: los estímulos que se otorguen derivados de la formación no son eficientes y suelen tener resultados negativos (Soto-Berenguel, 2003).

El profesional encargado de dirigir el talento humano debe tener una visión clara que le permita advertir de forma efectiva el comportamiento del personal interno, analizar de forma objetiva las relaciones laborales relacionadas entre su conducta y el área donde se desenvuelven, para prevenir anticipadamente alguna dificultad que se pueda ocasionar y poder intervenir a tiempo de forma efectiva.

La importancia que el personal se encuentre capacitado es un factor indispensable en la organización dando una gran relevancia a las herramientas usadas para medir el grado de conocimiento como son cuestionarios, entrevistas y otras herramientas que ayudan a conseguir información del estado actual del trabajador para un mejoramiento y por ende la obtención de los objetivos propuestos (Jurado-de-los-Santos, 2006). El futuro de las organizaciones depende de su formación estratégica, ya que garantiza controlar sus actividades de forma óptima, progresiva y ordenada, convirtiéndose en una herramienta capaz de ejecutar acciones orientadas a alcanzar metas y objetivos.

\section{Competencia laboral y evaluación de desempeño en el sector salud.}

Todo ser humano tiene derecho a la salud, constituyéndose en un factor insustituible y que interviene directamente en el bienestar de la social, desarrollo y economía de la población, factores preponderantes que comprometen a las instituciones hospitalarias a 


\section{CIENCIAMATRIA}

Revista Interdisciplinaria de Humanidades, Educación, Ciencia y Tecnología

Año VII. Vol. VII. N¹2. Enero - Junio. 2021

Hecho el depósito de ley: pp201602FA4721

ISSN-L: 2542-3029; ISSN: 2610-802X

Universidad Nacional Experimental Francisco de Miranda (UNEFM). Santa Ana de Coro. Venezuela

Karen Lisseth Apolo-Pérez; Juan Edmundo Álvarez-Gavilanes;

William Giovanni Vicuña-Matute

brindar atención oportuna de calidad, accesible para todas las personas y con calidez (Bernal-González, et al., 2014).

La integración de grupos de trabajo, puede durar meses o hasta años ya que es fundamental aprovechar sus capacidades y habilidades, los mismos que son importantes para el logro de objetivos de la organización, por lo cual el talento humano se convierte en el activo más importante de una administración, más aún cuando el servicio que brinda la organización está dirigida al sector salud. Cada talento humano posee característica y perfiles que hacen que su administración no sea una tarea fácil, ya que interfiere mucho la diversidad de aptitudes, compromisos y comportamientos además de los conocimientos especializados que se debe tener en el tema de salud dando un plus adicional a este talento (Bernal-González, et al., 2014).

Por lo tanto, es fundamental establecer responsabilidades y definir actividades a las personas que llevaran a cabo el proceso de gestión del talento humano, además debe tener acceso a los recursos técnicos, disponibilidad de inversión y espacios adecuados para que se trabaje efectivamente para la consecución de los objetivos, siendo el capital humano quien debe enfocar toda la atención de la empresa, ya que debe invertir mucho en su formación, incremento de capacidades y aptitudes que logren el mejoramiento del mismo para convertirse en un activo capaz de resolver sus propios problemas, establecerse objetivos y dar todo su esfuerzo por la organización, trabajando de manera activa y satisfactoria (Cordero, et al., 2019).

El proceso de gestión del talento humano está basado en competencias, quienes organizan el trabajo y lo distribuyen acorde a sus capacidades humanas, para la consecución de las metas y objetivos basados en los lineamientos formulados por el Sistema Nacional de Salud, siendo un proceso transversal y permanente para el desarrollo y sustento de las competencias, para brindar una atención integral de la población en función del logro de los resultados establecidos (Mejía-Giraldo, et al., 2012). La competencia laboral es el cumulo de conocimientos, habilidades, capacidades y actitudes que brindan la oportunidad de tratar temas relacionados con el trabajo. Se 


\section{CIENCIAMATRIA}

Revista Interdisciplinaria de Humanidades, Educación, Ciencia y Tecnología

Año VII. Vol. VII. N¹2. Enero - Junio. 2021

Hecho el depósito de ley: pp201602FA4721

ISSN-L: 2542-3029; ISSN: 2610-802X

Universidad Nacional Experimental Francisco de Miranda (UNEFM). Santa Ana de Coro. Venezuela

Karen Lisseth Apolo-Pérez; Juan Edmundo Álvarez-Gavilanes;

William Giovanni Vicuña-Matute

entiende que los conocimientos que presentan los trabajadores están en conjunto con capacidades y cualidades que ayudan a tomar decisiones y permite accionar según la competencia lo exige (Alonso-Suárez, et al., 2010).

Las competencias laborales de un puesto son fundamentales dentro de la gestión del recurso humano, ya que parte desde el inicio cómo es la selección de personal idóneo, capacitación constante, evaluación periódica, retribución y compensación dependiendo de las circunstancias. Por tal motivo surge la necesidad de investigación de las competencias dentro de la gestión del talento humano (Calderón-Hernández \& NaranjoValencia, 2004).

La competencia se crea bajo el concepto del comportamiento que se asocia con la generación de trabajos asignados, no da atención a los procesos grupales más bien relaciona la hoja de vida, capacitaciones enfocadas a conductas y tareas específicas (Chávez-Epiquén, et al., 2018). La competencia laboral es parte principal para lograr un enfoque integral, desde su concepción ya que debe relacionar el mundo laboral la sociedad, educación, enfocando en el aumento del talento humano como principio de conocimiento, competitividad e innovación (Ruiz de Vargas, 2005).

Para el crecimiento institucional es fundamental que cuenten con personal capacitado permitiendo definir metas y ampliar competencias que mejoren el desempeño y la productividad, haciendo más competitiva llegando a la satisfacción del cliente, usando como herramienta la evaluación del desempeño (Alveiro-Montoya, 2009). Gestionar de manera efectiva su desarrollo intelectual y que los mismos sean aplicados a las funciones que realicen, así como capacitaciones periódicas para el mejoramiento de las evaluaciones de desempeño.

Para asegurar resultados óptimos en la empresa, es indispensable que el trabajador adopte nuevas formas de conducta esto se logra a través de la evaluación continua para adoptar medidas inmediatas que corrijan esta situación, donde intervengan directamente el desempeño y la formación del empleado convirtiéndose en un sistema integrado de la gestión del talento humano (Alveiro-Montoya, 2009). 


\section{CIENCIAMATRIA}

Revista Interdisciplinaria de Humanidades, Educación, Ciencia y Tecnología

Año VII. Vol. VII. N¹2. Enero - Junio. 2021

Hecho el depósito de ley: pp201602FA4721

ISSN-L: 2542-3029; ISSN: 2610-802X

Universidad Nacional Experimental Francisco de Miranda (UNEFM). Santa Ana de Coro. Venezuela

Karen Lisseth Apolo-Pérez; Juan Edmundo Álvarez-Gavilanes;

William Giovanni Vicuña-Matute

Es importante evaluar de forma periódica las destrezas del personal, ya que esto permite tener pleno entendimiento de las debilidades de cada departamento para tomar medidas correctivas de manera inmediata, establecer controles de calidad y verificar el cumplimiento de metas a corto plazo de cada servicio, asegurando así el éxito de la empresa a través de la eficacia y eficiencia de los servicios que brinda (Manjarrés, et al., 2013).

Una de las principales tareas del área de gestión del talento humano es la evaluación constante del personal y sus comportamientos desarrollando estrategias que le permitan actuar de forma inmediata y efectiva en caso de controversias que afecten directamente al funcionamiento de la organización. Toda empresa con excelente gestión administrativa debe utilizar la técnica de evaluación de desempeño, ya que permite evaluar de forma periódica a los empleados para establecer la calidad del desempeño del trabajo midiendo la responsabilidad en sus labores (Capuano, 2004). Para lo antes expuesto nace la importancia de la evaluación periódica por parte de los directivos de una organización, además de una retroalimentación de sus resultados producto de sus esfuerzos (AlveiroMontoya, 2009).

Otro objetivo de la evaluación de desempeño es la de dar a conocer al personal cómo es su situación en su entorno laboral y lo que la empresa espera de ellos, incentivar los méritos y el cumplimiento de los objetivos, corrección oportuna de actitudes o comportamientos que interfieran en el desarrollo de un proceso, además se logra que el personal evaluado pueda verse a sí mismo en un futuro haciendo una retrospectiva de su vida laboral analizando sus fortalezas y debilidades de cada empelado. La evaluación periódica de la gestión del talento humano es importante y debe ser una de las prioridades de toda organización, ya que vincula y cuantifica una serie de conceptos enfocados a la administración efectiva del talento humano (López-Puig, et al., 2017).

Existen múltiples opciones que las empresas usan con la finalidad de contabilizar las aptitudes de los trabajadores y sea supervisado por el jefe de área o algún superior que este ambientado al rendimiento, autoevaluación, donde el propio empleado mide su 


\section{CIENCIAMATRIA}

Revista Interdisciplinaria de Humanidades, Educación, Ciencia y Tecnología

Año VII. Vol. VII. N¹2. Enero - Junio. 2021

Hecho el depósito de ley: pp201602FA4721

ISSN-L: 2542-3029; ISSN: 2610-802X

Universidad Nacional Experimental Francisco de Miranda (UNEFM). Santa Ana de Coro. Venezuela

Karen Lisseth Apolo-Pérez; Juan Edmundo Álvarez-Gavilanes;

William Giovanni Vicuña-Matute

desempeño sugiriendo nuevos mecanismos de mejoramiento entre compañeros o cargos con funciones similares donde valoran a sus jefes superiores; otra parte fundamental es la evaluación del cliente a los empleados con los que interactúan, otro modelo es la conocida como 360 grados la cual involucra todos los métodos anteriores la misma que resulta muy compleja, demanda tiempo y análisis detallado donde cada usuario interno es supervisado de forma tecnológica, contando siempre con su autorización (RiveroRamírez, 2019).

La mayoría de organizaciones son capaces de medir la gestión de desempeño de su personal, algunos trabajan de forma organizada proponiendo condiciones óptimas para la evaluación del potencial y talento (Gutiérrez-Alvarado, 2011). La medición periódica de los resultados de eficiencia y eficacia, así como las habilidades que poseen el personal constituye los indicadores de la gestión necesaria en toda empresa, siendo una herramienta que permite comparar resultados en base de objetivos planteados contra los obtenidos (Pérez \& Vera Méndez, 2011).

\section{Clima organizacional en el Sector Salud y su modelo de gestión}

El clima organizacional de una empresa dependiendo de su naturaleza puede ser adecuada o convertirse en un problema que afectara al desenvolvimiento de la empresa. La incorporación de varias características que delimitan el ambiente de trabajo de una empresa se conoce como clima laboral, y enfoca las actitudes, comportamiento y relaciones del personal que conforma el talento humano.

Analizar en las instituciones públicas de salud el clima laboral de los empleados es una de las herramientas que contribuyen para mejorar, ya que a partir de su estudio se

establece los puntos claves que deben ser atendidas con inmediatez para mejorar la calidad de la vida laboral y conjuntamente el mejoramiento de los servicios de salud que se brinda a la población. El descrito anteriormente influye de forma significativa en el desempeño de los empleados, mejorando la productividad y el comprometimiento de los empleados con la organización (Bernal-González, et al., 2014). 


\section{CIENCIAMATRIA}

Revista Interdisciplinaria de Humanidades, Educación, Ciencia y Tecnología

Año VII. Vol. VII. N¹2. Enero - Junio. 2021

Hecho el depósito de ley: pp201602FA4721

ISSN-L: 2542-3029; ISSN: 2610-802X

Universidad Nacional Experimental Francisco de Miranda (UNEFM). Santa Ana de Coro. Venezuela

Karen Lisseth Apolo-Pérez; Juan Edmundo Álvarez-Gavilanes;

William Giovanni Vicuña-Matute

La motivación al personal es un factor importante para un clima laboral adecuado en la institución, si el personal está motivado le permitirá generar en un ambiente agradable para trabajar de manera óptima, pero si el ambiente laboral no es agradable, no podrán desarrollar sus habilidades. Una de la inconformidad de los trabajadores es la remuneración, para que el personal se motive es necesario que perciba una remuneración acorde a sus responsabilidades, ya que se sentirá comprometido con el trabajo y se reflejará recompensado su esfuerzo, ya que muchas de sus necesidades son de forma material (Miranda-Hoyes, 2016).

Parte de las aptitudes del personal se percibe mejor cuando este se desenvuelve dentro de un ambiente óptimo.

Una de las grandes preocupaciones de las administraciones actuales es contar con personal desmotivado, incorrectamente retribuido acorde a sus responsabilidades, generando un pésimo clima laboral, afectando a la productividad de la organización y acrecentándose con el paso del tiempo, convirtiéndose en una preocupación de las gerencias de hoy en día (Valencia, et al., 2019). En el desarrollo organizativo se enfoca a la organización sus necesidades y prioridades fundamentos. En el desarrollo de la trayectoria profesional, señala la preparación y su importancia dentro de su carrera profesional. Dentro de lo que cabe el desarrollo personal se consideran las capacidades interpersonales y el crecimiento individual.

Se debe preparar de forma eficaz al trabajador para su puesto asignado, entender sus aspiraciones y objetivos esperados, advertir debilidades que se pueden corregir para que puedan desenvolverse de forma eficiente en su trabajo, mejoramiento de las relaciones interpersonales que permitan dar referencias para posibles candidatos para incrementos de salarios entre otros (Rivero-Ramírez, 2019). El descontento del usuario, es producto de la desatención del área del talento humano que no está aprovechando de forma efectiva el potencial que posee cada uno, no permitiendo el desarrollo de sus capacidades sin que puedan sobresalir. 


\section{CIENCIAMATRIA}

Revista Interdisciplinaria de Humanidades, Educación, Ciencia y Tecnología

Año VII. Vol. VII. N¹2. Enero - Junio. 2021

Hecho el depósito de ley: pp201602FA4721

ISSN-L: 2542-3029; ISSN: 2610-802X

Universidad Nacional Experimental Francisco de Miranda (UNEFM). Santa Ana de Coro. Venezuela

Karen Lisseth Apolo-Pérez; Juan Edmundo Álvarez-Gavilanes;

William Giovanni Vicuña-Matute

Aunque los trabajadores se gestionan de forma autónoma, ellos son los llamados a demostrar lo mejor de sus actitudes para sobresalir sobre el resto (Rivero \& Dabos, 2017). El poco compromiso y preocupación por el mejoramiento del desempeño de cada trabajador afecta el desempeño laboral y por ende influye en la atención del usuario y su aceptación o no de los servicios recibidos. La poca atención a los procesos de desarrollo laboral influye en el desarrollo laboral, rendimiento y productividad que se ofrece a los clientes (Matabajoy-Montilla, et al., 2017).

El mejoramiento del clima laboral depende de la gestión efectiva del talento humano, ya que es responsable de generar espacios de trabajo agradable, donde se potencien los conocimientos de las personas, se sientan satisfechos con sus funciones y conforme su salario, asegurando la productividad de la empresa (Vera-Barbosa \& Blanco-Ariza, 2019). Todo este conjunto de la gestión organizacional hará que el personal desarrolle sus habilidades y capacidades dentro de su entorno técnico, creando un ambiente óptimo para el intercambio de ideas, conocimientos, trabajo en equipo logrando que alcance un nivel de desempeño necesario y eficiente capaz de resolver problemas, búsqueda de soluciones inmediatas, así como ser precisos con la toma de decisiones creando verdaderos líderes en cada puesto de trabajo. (Espín-Oleas, et al., 2015).

Establecer programas de premios, ascensos y bonos por su desempeño o metas cumplidas en determinados tiempos. Todos estos recursos empleados para lograr lo establecido anteriormente es indispensable que sean administrados correctamente, ya que son fundamentales para cumplir con las metas trazadas (Espín-Oleas, et al., 2015). Así mismo, se señalan varios modelos referentes al desempeño del talento humano según indica (Montenegro, 2007, p. 33-40).

El modelo brasilero clasificó al profesional de carrera en los siguientes niveles:

Básico: se refiere al personal que cuenta con una carrera profesional, realiza cursos de capacitación e inducción, y certificados.

Competente: se refiere al personal organizado por nivel óptimo, clasificación, premios y promoción. 


\section{CIENCIAMATRIA \\ Revista Interdisciplinaria de Humanidades, Educación, Ciencia y Tecnología \\ Año VII. Vol. VII. N¹2. Enero - Junio. 2021 \\ Hecho el depósito de ley: pp201602FA4721 \\ ISSN-L: 2542-3029; ISSN: 2610-802X \\ Universidad Nacional Experimental Francisco de Miranda (UNEFM). Santa Ana de Coro. Venezuela}

Karen Lisseth Apolo-Pérez; Juan Edmundo Álvarez-Gavilanes;

William Giovanni Vicuña-Matute

Sobresaliente: tiene certificación avanzada en un área específica y promoción.

El modelo cubano realiza actividades relacionadas con la superación profesional y trabajo metodológico en equipo.

Ejecución: exige la participación de profesionales en actividades diseñadas para un área específica.

Evaluación: constante de los cambios basados en el desempeño enfocado en las competencias asignadas.

El modelo chileno se basa en patrones cuya base es la responsabilidad que asegura los servicios del ámbito profesional que ayudan a cumplir expectativas del entorno social relacionadas con el incremento del desempeño usando como herramienta de control del cumplimiento de sus objetivos a través de la evaluación.

El modelo del Reino Unido evalúa basándose en la formación y experiencia los perfiles que cuentan los profesionales, así como también sus resultados contrastándolos entre si y los usuarios externos.

Parte de la estrategia utilizada para motivar a los usuarios es formando un vínculo entre ellos que permitan un ambiente óptimo entre sí.

El modelo de los Estados Unidos prepara a los profesionales, hace un análisis de su entorno, se preocupa por su formación, observa las responsabilidades asignadas relacionadas con su profesión.

El modelo colombiano revisa el desarrollo pedagógico acorde al progreso de las capacidades relacionadas con el aprendizaje, tecnológicas y la tecnología.

En la parte del desarrollo profesional se realiza un análisis de los niveles alcanzados desde el más alto dentro de la competencia profesional.

El objetivo de esta investigación se centró en el mejoramiento del modelo de gestión estratégica actual de talento humano, orientadas a la formación del personal con el que cuenta el HGM usándolo como un instrumento que permita planificar de manera organizativa las capacitaciones continuas según su área de trabajo y actividades, analizando el perfil de cada servidor acorde a sus aptitudes, capacidades y preparación 
CIENCIAMATRIA

Revista Interdisciplinaria de Humanidades, Educación, Ciencia y Tecnología

Año VII. Vol. VII. N¹2. Enero - Junio. 2021

Hecho el depósito de ley: pp201602FA4721

ISSN-L: 2542-3029; ISSN: 2610-802X

Universidad Nacional Experimental Francisco de Miranda (UNEFM). Santa Ana de Coro. Venezuela

Karen Lisseth Apolo-Pérez; Juan Edmundo Álvarez-Gavilanes;

William Giovanni Vicuña-Matute

profesional para aprovechar estos recursos y mejorar la gestión hospitalaria de forma efectiva.

\section{MÉTODO}

El alcance de esta investigación es descriptivo - transversal, debido a que en el desarrollo de esta propuesta se definió la relación que existe entre las variables tanto de formación académica del personal y desempeño del talento humano, para proponer una propuesta que optimice la gestión estratégica del talento humano para el mejoramiento de la operatividad de la organización, recopilándose la información y descripción de eventos que sucedieron en un tiempo establecido y estuvo dirigida al personal que labora en la institución.

La técnica utilizada para la recopilación de información fue la encuesta online, mientras que el instrumento fue de tipo cuestionario de 13 ítems con varias alternativas de respuestas, el cual permitió obtener y analizar la información levantada. La información recopilada fue analizada en razón de la estadística descriptiva, presentándose a través de porcentajes y frecuencias. Siendo la muestra poblacional conformada por 192 servidores del Hospital General de Machala (HGM), realizándose un muestreo por conveniencia, que es una técnica no probabilística elaborada de manera aleatoria formado por la disponibilidad de los servidores que formaron parte de la investigación (Rodríguez, et al., 2019).

\section{RESULTADOS}

De la investigación realizada, entre los principales resultados que se obtuvieron en la aplicación de la encuesta son los siguientes:

En lo referente a las competencias laborales el 59,9\% de los encuestados manifestó que, al ingresar a la institución, la unidad de talento humano no le designó las funciones a desempeñar, por otra parte, tenemos que el $52,6 \%$ del personal indicó que las actividades que desempeña no están acorde al perfil profesional que ellos poseen, estos dos puntos 


\section{CIENCIAMATRIA}

Revista Interdisciplinaria de Humanidades, Educación, Ciencia y Tecnología

Año VII. Vol. VII. N¹2. Enero - Junio. 2021

Hecho el depósito de ley: pp201602FA4721

ISSN-L: 2542-3029; ISSN: 2610-802X

Universidad Nacional Experimental Francisco de Miranda (UNEFM). Santa Ana de Coro. Venezuela

Karen Lisseth Apolo-Pérez; Juan Edmundo Álvarez-Gavilanes;

William Giovanni Vicuña-Matute

derivan que el $36,5 \%$ de talento humano no se encuentre satisfecho con las actividades que desempeña en su puesto de trabajo.

Otra valoración importante de la encuesta es la evaluación de desempeño, donde un $69,80 \%$ de los encuestados tienen la apreciación de una mala estructuración y aplicación de la evaluación, donde indican que no se apega con su realidad laboral.

Cabe indica que el $66,7 \%$ de los servidores de la institución considera que su remuneración salarial no está acorde a las actividades o funciones que realizan, añadiendo que un $87 \%$ del personal no se encuentra motivado por su desempeño originando un clima laboral desfavorable.

Los resultados obtenidos referente a la capacitación de los servidores serán presentados en el paso uno de esta propuesta.

\section{PROPUESTA}

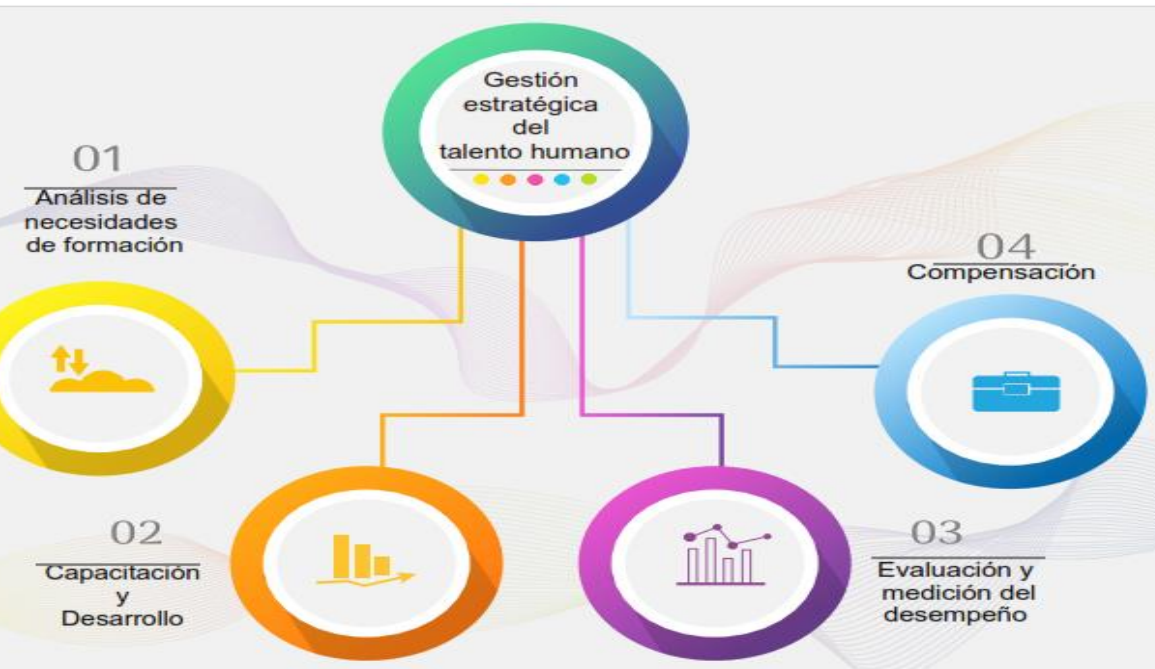

Figura 1. Esquema de la propuesta. 


\section{CIENCIAMATRIA}

Revista Interdisciplinaria de Humanidades, Educación, Ciencia y Tecnología

Año VII. Vol. VII. N¹2. Enero - Junio. 2021

Hecho el depósito de ley: pp201602FA4721

ISSN-L: 2542-3029; ISSN: 2610-802X

Universidad Nacional Experimental Francisco de Miranda (UNEFM). Santa Ana de Coro. Venezuela

Karen Lisseth Apolo-Pérez; Juan Edmundo Álvarez-Gavilanes;

William Giovanni Vicuña-Matute

La propuesta del mejoramiento de la gestión del talento humano del HGM, establece un esquema de cuatro etapas secuenciales, donde se detalla el contenido de cada una estableciendo parámetros que deben ser llevado de forma organizada orientadas a analizar las necesidades de formación del personal de cada departamento.

\section{Análisis de necesidades de formación}

Una vez aplicada la encuesta al talento humano del HGM, se toma como base fundamental los resultados obtenidos haciendo énfasis en la deficiencia existente del conocimiento específico y su aplicación en sus puestos de trabajo, dando un punto de inicio para diagnosticar, analizar y dimensionar las falencias que posee el personal.

Con los resultados obtenidos se evidencia que el $58.9 \%$ de los encuestados no recibieron una inducción adecuada para el desempeño de sus funciones en el área asignada, otro $76.60 \%$ desconoce sobre la existencia de programas de capacitación en la institución, un $52.10 \%$ manifiesta que no ha recibido capacitaciones acordes a la función que desempeñan.

Una vez diagnosticado los resultados de la encuesta, se concluye que, deben aplicarse capacitaciones específicas del personal, dirigido al área donde desarrollan sus actividades.

Para mejorar esta deficiencia se propone el uso de un formulario que sea aplicado a todas las áreas de la institución, que permita identificar necesidades de capacitación enfocada a áreas específicas. La herramienta propuesta ayudará a la unidad de talento humano a conocer la realidad y necesidades exactas que requiere el personal. 


\section{CIENCIAMATRIA}

Revista Interdisciplinaria de Humanidades, Educación, Ciencia y Tecnología

Año VII. Vol. VII. N¹2. Enero - Junio. 2021

Hecho el depósito de ley: pp201602FA4721

ISSN-L: 2542-3029; ISSN: 2610-802X

Universidad Nacional Experimental Francisco de Miranda (UNEFM). Santa Ana de Coro. Venezuela

Karen Lisseth Apolo-Pérez; Juan Edmundo Álvarez-Gavilanes;

William Giovanni Vicuña-Matute

\section{Tabla1.}

Identificación de necesidades de formación.

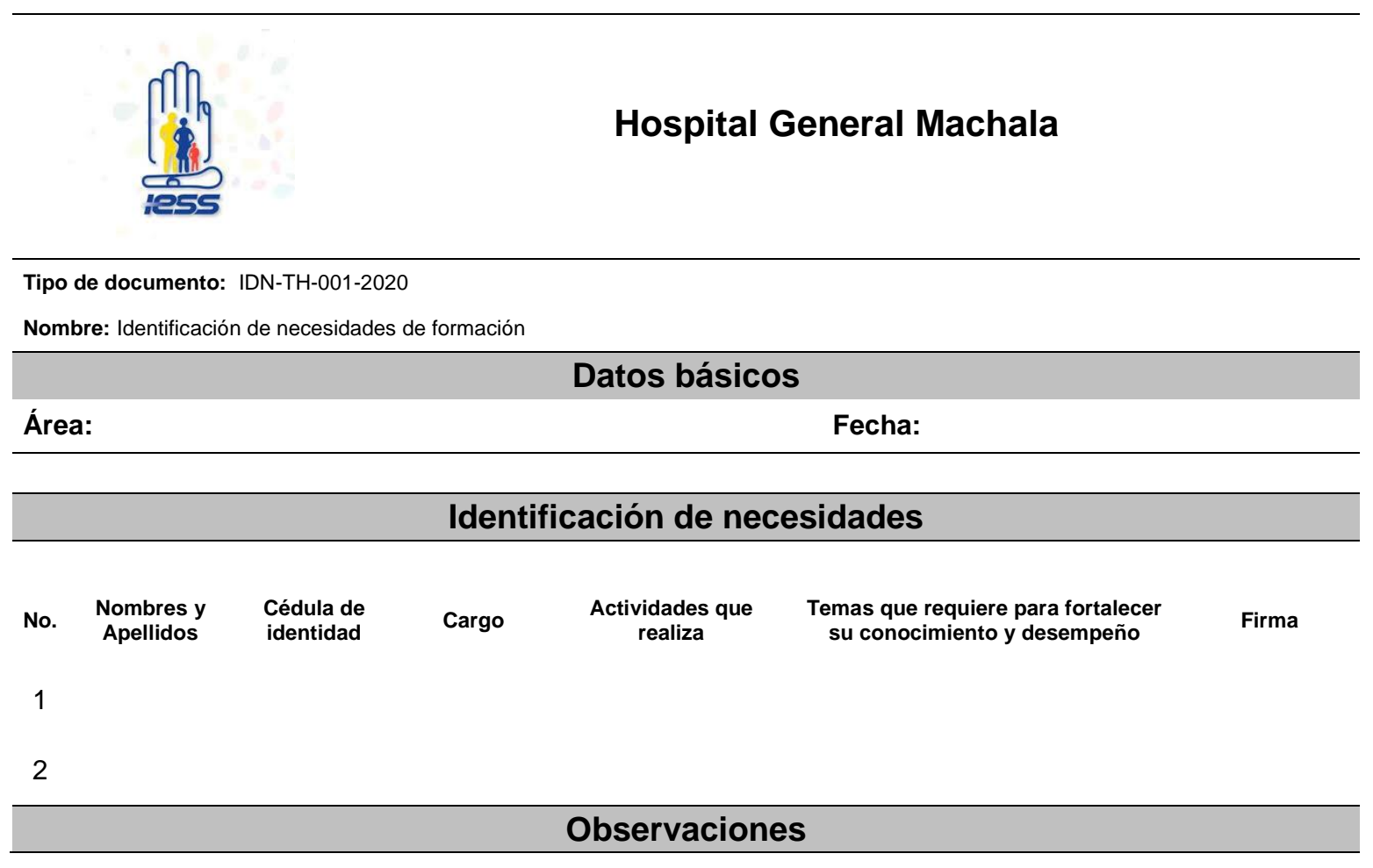

Fuente: Autores.

\section{Capacitación y desarrollo}

Una vez concluido el análisis de necesidades de formación, aplicando el respectivo formulario que recopilará la información específica para identificar las necesidades del personal, se procede a elaborar un cronograma de capacitación con temas relacionados en las actividades que realizan en sus puestos de trabajo, este programa debe ser planificado y sustentado en base a los resultados obtenidos, este plan debe ser programado y gestionado económicamente a través del plan anual de contratación institucional a fin de asegurar su ejecución de manera efectiva.

Este estudio se ha obtenido mediante reuniones de trabajo con los coordinadores de área del HGM referente a necesidades de capacitación del personal que tienen a su cargo, 


\section{CIENCIAMATRIA}

Revista Interdisciplinaria de Humanidades, Educación, Ciencia y Tecnología

Año VII. Vol. VII. N¹2. Enero - Junio. 2021

Hecho el depósito de ley: pp201602FA4721

ISSN-L: 2542-3029; ISSN: 2610-802X

Universidad Nacional Experimental Francisco de Miranda (UNEFM). Santa Ana de Coro. Venezuela

Karen Lisseth Apolo-Pérez; Juan Edmundo Álvarez-Gavilanes;

William Giovanni Vicuña-Matute

arrojando resultados que permiten cuantificar la necesidad de formación del personal de cada servicio para establecer prioridades al momento de impartir la inducción respectiva. En base a las necesidades recopiladas en la tabla 2 se establece el siguiente cronograma de capacitación para las áreas del HGM.

\section{Tabla 2.}

Cronograma de capacitación.

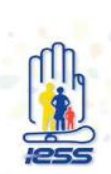

Cronograma de capacitación

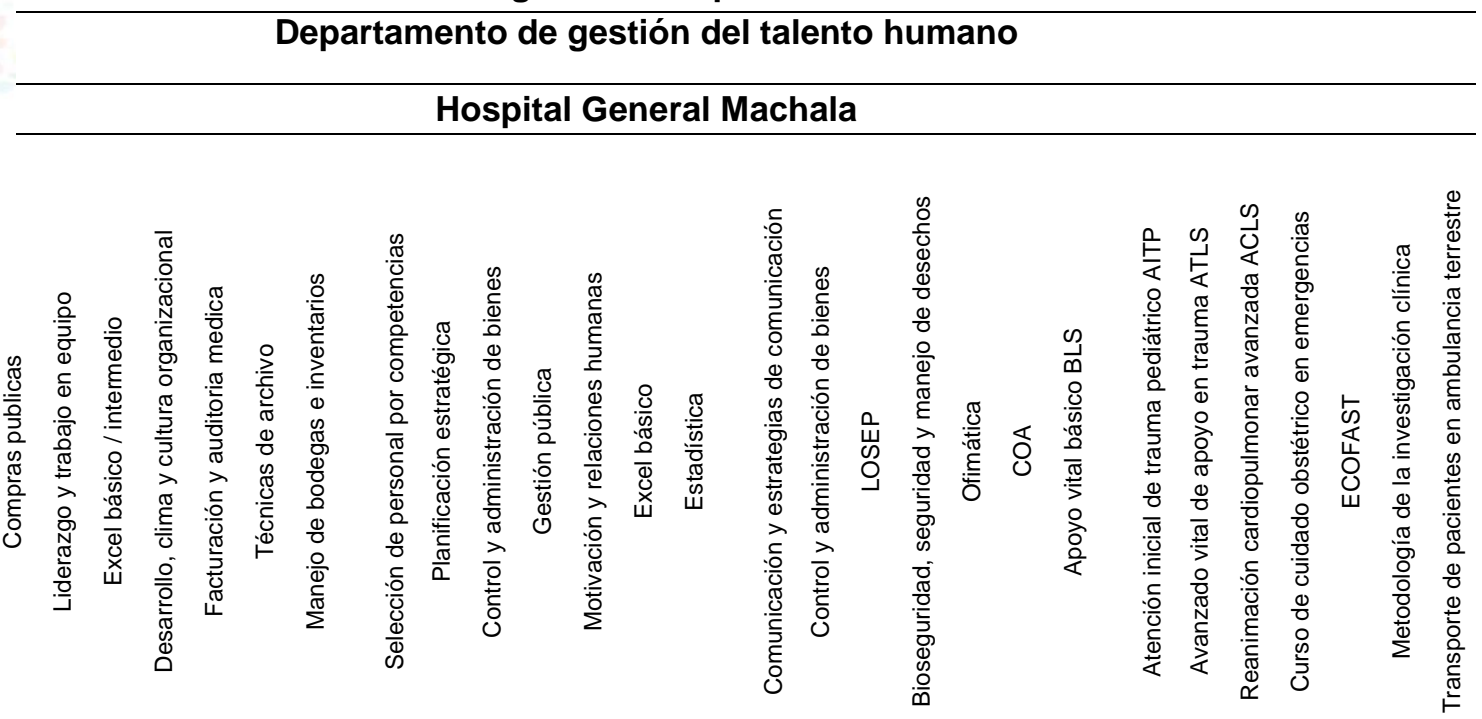

ÁREAS

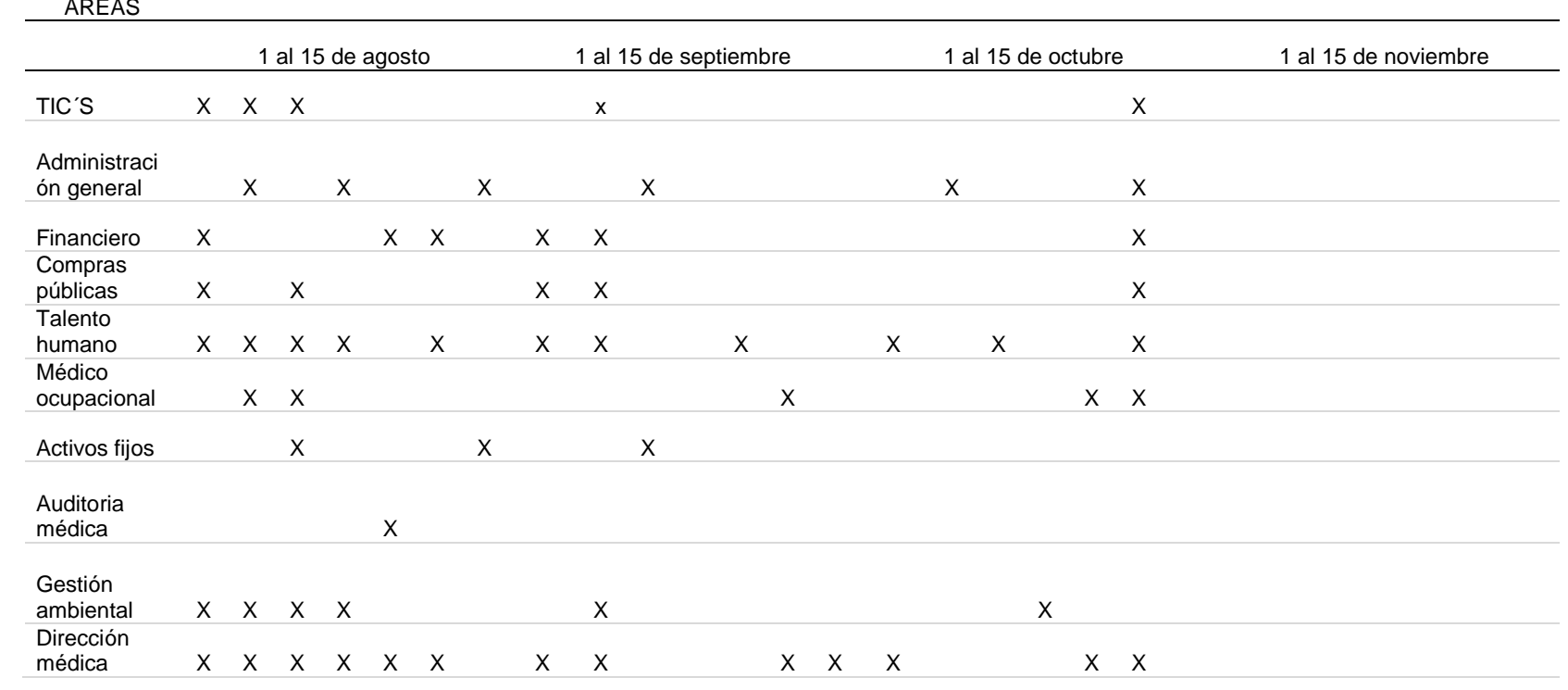




\section{CIENCIAMATRIA}

Revista Interdisciplinaria de Humanidades, Educación, Ciencia y Tecnología

Año VII. Vol. VII. N¹2. Enero - Junio. 2021

Hecho el depósito de ley: pp201602FA4721

ISSN-L: 2542-3029; ISSN: 2610-802X

Universidad Nacional Experimental Francisco de Miranda (UNEFM). Santa Ana de Coro. Venezuela

Karen Lisseth Apolo-Pérez; Juan Edmundo Álvarez-Gavilanes;

William Giovanni Vicuña-Matute

\begin{tabular}{|c|c|c|c|c|c|c|c|c|c|c|c|c|c|c|c|c|c|c|c|c|c|c|c|c|c|c|c|c|}
\hline $\begin{array}{l}\text { Unidad } \\
\text { jurídica }\end{array}$ & & & & & & & & & & & $x$ & & & & & $x$ & $x$ & & $x$ & & & & & & & & & \\
\hline $\begin{array}{l}\text { Seguimiento } \\
\text { y control }\end{array}$ & & & & $x$ & $x$ & & & & & & & & & & & & & & $x$ & & & & & & & & & \\
\hline Enfermería & & & & & & & & & & & & & & & & & & & & & $\mathrm{X}$ & $X$ & $X$ & $X$ & $X$ & $x$ & $\mathrm{X}$ & $X$ \\
\hline Total & 6 & 6 & 7 & 5 & 4 & 3 & 2 & 4 & 6 & 2 & 1 & 1 & 2 & 1 & 2 & 2 & 2 & 1 & 4 & 7 & 1 & 1 & 1 & 1 & 1 & 1 & 1 & 1 \\
\hline
\end{tabular}

Fuente: Unidad de talento humano del HGM.

\section{Evaluación y medición del desempeño}

Con fecha 07 de marzo del 2018 mediante acuerdo ministerial No. MDT-2018-0041 el ministerio de trabajo expidió la norma técnica del subsistema de evaluación del desempeño, además aprobó el instructivo para el registro de información en los formatos de evaluación del desempeño.

Sin embargo, como una alternativa se propone un formulario flexible a los cambios institucionales, gestionado de manera interna que se adecúe a la realidad de cada departamento, permitiendo evaluar de forma eficiente el desempeño individual y grupal, entendiendo que los objetivos planteados por la organización se definen por área, por tal motivo los premios establecidos se realizarán de manera grupal para fortalecer el compromiso y trabajo en equipo como se observa en la tabla 3. 


\section{CIENCIAMATRIA}

Revista Interdisciplinaria de Humanidades, Educación, Ciencia y Tecnología

Año VII. Vol. VII. N¹2. Enero - Junio. 2021

Hecho el depósito de ley: pp201602FA4721

ISSN-L: 2542-3029; ISSN: 2610-802X

Universidad Nacional Experimental Francisco de Miranda (UNEFM). Santa Ana de Coro. Venezuela

Karen Lisseth Apolo-Pérez; Juan Edmundo Álvarez-Gavilanes;

William Giovanni Vicuña-Matute

\section{Tabla 3.}

Cuadro operativo de medición de desempeño.

\section{Cuadro operativo de medición de desempeño del Hospital General Machala}

\begin{tabular}{|c|c|c|c|c|c|c|c|c|c|c|c|c|}
\hline & \multirow[t]{2}{*}{$\begin{array}{l}\text { \& } \\
\stackrel{0}{0} \\
\text { ㅎํ }\end{array}$} & \multirow[t]{2}{*}{$\frac{\stackrel{0}{0}}{0}$} & \multirow[t]{2}{*}{ 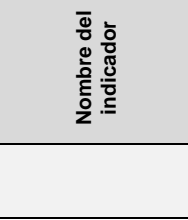 } & \multirow[t]{2}{*}{ 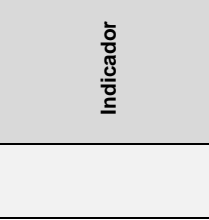 } & \multirow[t]{2}{*}{ 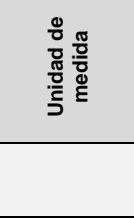 } & \multirow[t]{2}{*}{ 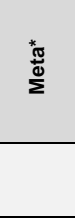 } & \multicolumn{4}{|c|}{ 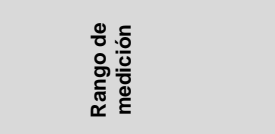 } & \multirow[t]{2}{*}{ 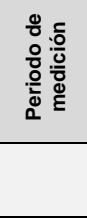 } & \multirow[t]{2}{*}{ 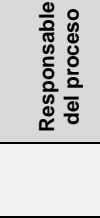 } \\
\hline & & & & & & & 인항 & & 幽 & 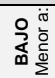 & & \\
\hline 1 & Capacitación & $\begin{array}{l}\text { Controlar } \\
\text { cumplimiento } \\
\text { plan anual de } \\
\text { capacitación }\end{array}$ & $\begin{array}{l}\text { Cumplimiento } \\
\text { planificación } \\
\text { anual }\end{array}$ & $\begin{array}{l}\text { Capacitaciones } \\
\text { realizadas/Capaci } \\
\text { taciones } \\
\text { Planificadas*100 }\end{array}$ & Porcentaje & $90 \%$ & $90 \%$ & $\begin{array}{l}90 \\
\%\end{array}$ & $\begin{array}{l}70 \\
\%\end{array}$ & $\begin{array}{l}70 \\
\%\end{array}$ & Anual & $\begin{array}{l}\text { Talento } \\
\text { Humano }\end{array}$ \\
\hline 2 & Capacitación & $\begin{array}{l}\text { Verificar } \mathrm{N}^{\circ} \text { de } \\
\text { empleados } \\
\text { capacitados }\end{array}$ & $\begin{array}{l}\text { Cumplimiento } \\
\text { de capacitación }\end{array}$ & $\begin{array}{l}\mathrm{N}^{\circ} \text { Empleados } \\
\text { capacitados } / \mathrm{N}^{\circ} \\
\text { total } \\
\text { empleados }{ }^{*} 100\end{array}$ & Porcentaje & $100 \%$ & $100 \%$ & $\begin{array}{l}90 \\
\%\end{array}$ & $\begin{array}{l}70 \\
\%\end{array}$ & $\begin{array}{l}65 \\
\%\end{array}$ & Anual & $\begin{array}{l}\text { Talento } \\
\text { Humano }\end{array}$ \\
\hline 3 & Capacitación & $\begin{array}{l}\text { Evaluar } \\
\text { satisfacción de } \\
\text { empleados } \\
\text { frente a } \\
\text { capacitaciones } \\
\text { recibidas }\end{array}$ & $\begin{array}{l}\text { Índice de } \\
\text { satisfacción de } \\
\text { empleados }\end{array}$ & $\begin{array}{l}\text { Tabulación de } \\
\text { encuestas de } \\
\text { reacción }\end{array}$ & Porcentaje & $100 \%$ & $100 \%$ & $\begin{array}{l}90 \\
\%\end{array}$ & $\begin{array}{l}70 \\
\%\end{array}$ & $\begin{array}{l}65 \\
\%\end{array}$ & Anual & $\begin{array}{l}\text { Talento } \\
\text { Humano }\end{array}$ \\
\hline 4 & $\begin{array}{l}\text { Gestión del } \\
\text { desempeño }\end{array}$ & $\begin{array}{l}\text { Medir el nivel } \\
\text { de ejecución de } \\
\text { un determinado } \\
\text { proceso }\end{array}$ & $\begin{array}{l}\text { Índice de } \\
\text { eficiencia }\end{array}$ & $\begin{array}{l}\text { Nro. procesos } \\
\text { realizados/Nro. } \\
\text { de empleados del } \\
\text { departamento }\end{array}$ & Porcentaje & $85 \%$ & $85 \%$ & $\begin{array}{l}85 \\
\%\end{array}$ & $\begin{array}{l}65 \\
\%\end{array}$ & $\begin{array}{l}65 \\
\%\end{array}$ & Anual & $\begin{array}{l}\text { Talento } \\
\text { Humano }\end{array}$ \\
\hline 5 & $\begin{array}{l}\text { Gestión del } \\
\text { desempeño }\end{array}$ & $\begin{array}{l}\text { Medir la } \\
\text { capacidad de } \\
\text { alcanzar los } \\
\text { resultados } \\
\text { esperados }\end{array}$ & $\begin{array}{l}\text { Índice de } \\
\text { eficacia }\end{array}$ & $\begin{array}{l}\text { Nro. de metas } \\
\text { establecidas/Nro. } \\
\text { de metas } \\
\text { cumplidas }\end{array}$ & Porcentaje & $95 \%$ & $95 \%$ & $\begin{array}{l}95 \\
\%\end{array}$ & $\begin{array}{l}75 \\
\%\end{array}$ & $\begin{array}{l}75 \\
\%\end{array}$ & Anual & $\begin{array}{l}\text { Talento } \\
\text { Humano }\end{array}$ \\
\hline 6 & $\begin{array}{l}\text { Gestión del } \\
\text { desempeño }\end{array}$ & $\begin{array}{l}\text { Medir los logros } \\
\text { de los objetivos } \\
\text { propuestos por } \\
\text { el hospital }\end{array}$ & $\begin{array}{l}\text { Índice de } \\
\text { efectividad }\end{array}$ & $\begin{array}{l}\text { Nro. de } \\
\text { resultados } \\
\text { logrados/Nro. de } \\
\text { resultados } \\
\text { proyectados }\end{array}$ & Porcentaje & $90 \%$ & $90 \%$ & $\begin{array}{l}90 \\
\%\end{array}$ & $\begin{array}{l}60 \\
\%\end{array}$ & $\begin{array}{l}60 \\
\%\end{array}$ & Anual & $\begin{array}{l}\text { Talento } \\
\text { Humano }\end{array}$ \\
\hline
\end{tabular}

Fuente: Arellano - Narváez (2016). 


\section{CIENCIAMATRIA}

Revista Interdisciplinaria de Humanidades, Educación, Ciencia y Tecnología

Año VII. Vol. VII. N¹2. Enero - Junio. 2021

Hecho el depósito de ley: pp201602FA4721

ISSN-L: 2542-3029; ISSN: 2610-802X

Universidad Nacional Experimental Francisco de Miranda (UNEFM). Santa Ana de Coro. Venezuela

Karen Lisseth Apolo-Pérez; Juan Edmundo Álvarez-Gavilanes;

William Giovanni Vicuña-Matute

Tabla 4.

Indicadores de medición de desempeño del HGM.

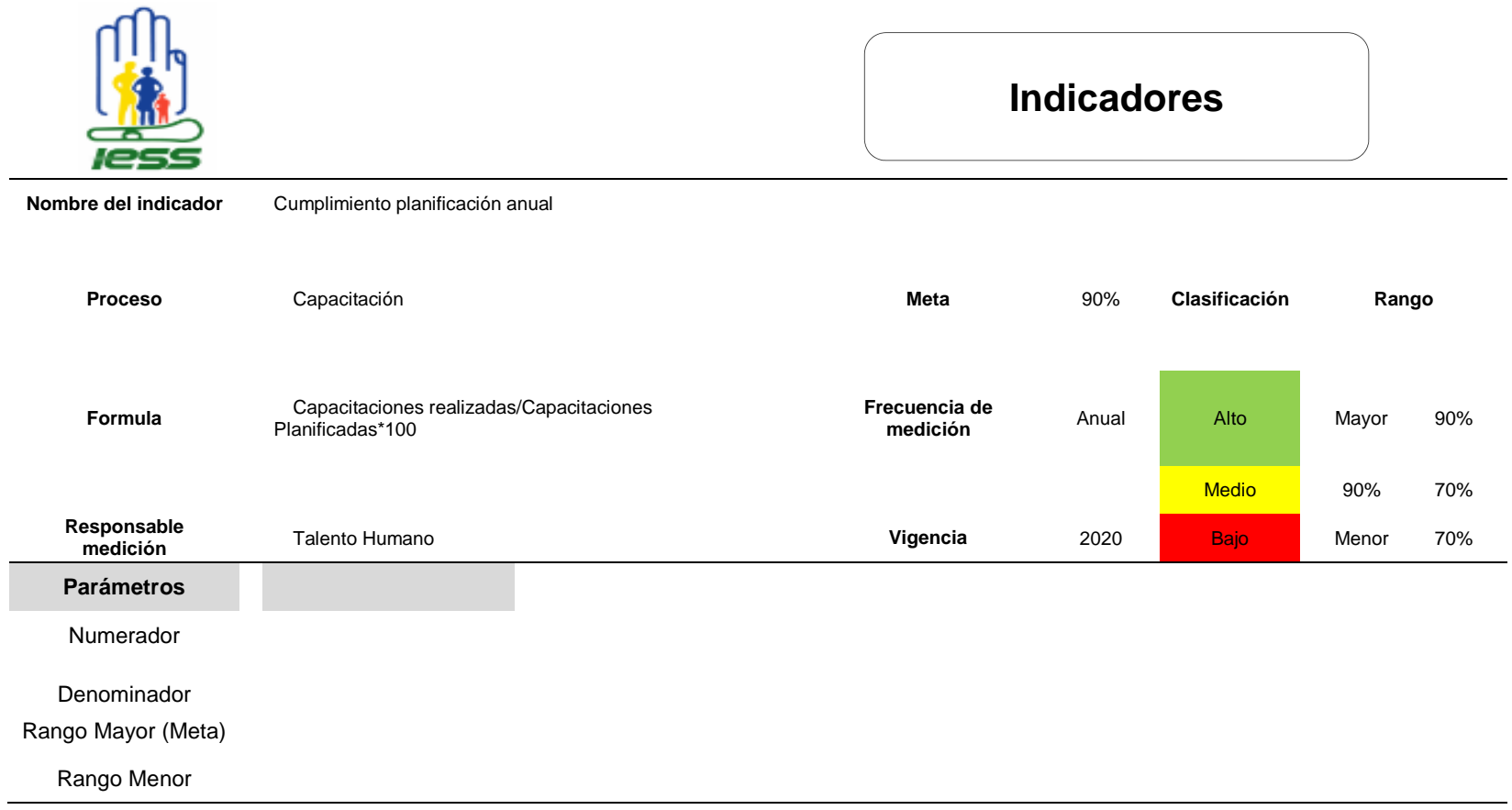

Fuente: Arellano - Narváez (2016).

\section{Compensación}

En base a los resultados obtenidos en la evaluación y medición de desempeño de las áreas, se ha tomado los tres porcentajes más altos, para que estos sean recompensados públicamente en el día de la seguridad social a través de reconocimientos y medallas para todo el departamento, valorando el esfuerzo y compromiso. 


\section{CIENCIAMATRIA}

Revista Interdisciplinaria de Humanidades, Educación, Ciencia y Tecnología

Año VII. Vol. VII. N¹2. Enero - Junio. 2021

Hecho el depósito de ley: pp201602FA4721

ISSN-L: 2542-3029; ISSN: 2610-802X

Universidad Nacional Experimental Francisco de Miranda (UNEFM). Santa Ana de Coro. Venezuela

Karen Lisseth Apolo-Pérez; Juan Edmundo Álvarez-Gavilanes;

William Giovanni Vicuña-Matute

\section{Tabla 4.}

Consolidación de calificación de desempeño.

\begin{tabular}{lc}
\hline Áreas calificadas & $\%$ \\
\hline Coordinar/a general de servicios clínicos & $\mathbf{9 7 , 7 3}$ \\
Coordinador/a general de servicios quirúrgicos & $\mathbf{9 7 , 4 2}$ \\
Subdirector administrativo financiero & $\mathbf{9 6 , 8 6}$ \\
Gerencia general & 96,39 \\
Coordinación de talento humano & 96,25 \\
Director técnico & 96,31 \\
Subdirección de apoyo diagnóstico y terapéutico & 96,24 \\
Coordinador/a financiero & 96,12 \\
Coordinación de farmacia & 95,32 \\
Subdirector administrativo financiero & 95,14 \\
Coordinador/a de adquisiciones & 94,68 \\
Subdirección de enfermería & 94,25 \\
Subdirección de enfermería & 93,84 \\
Subdirección técnica de medicina critica & 93,60 \\
Subdirección técnica medica & 92,37 \\
\hline
\end{tabular}

Fuente: Informe consolidado de resultados/IN-GEP-02-02 FOR-10/ HGM.

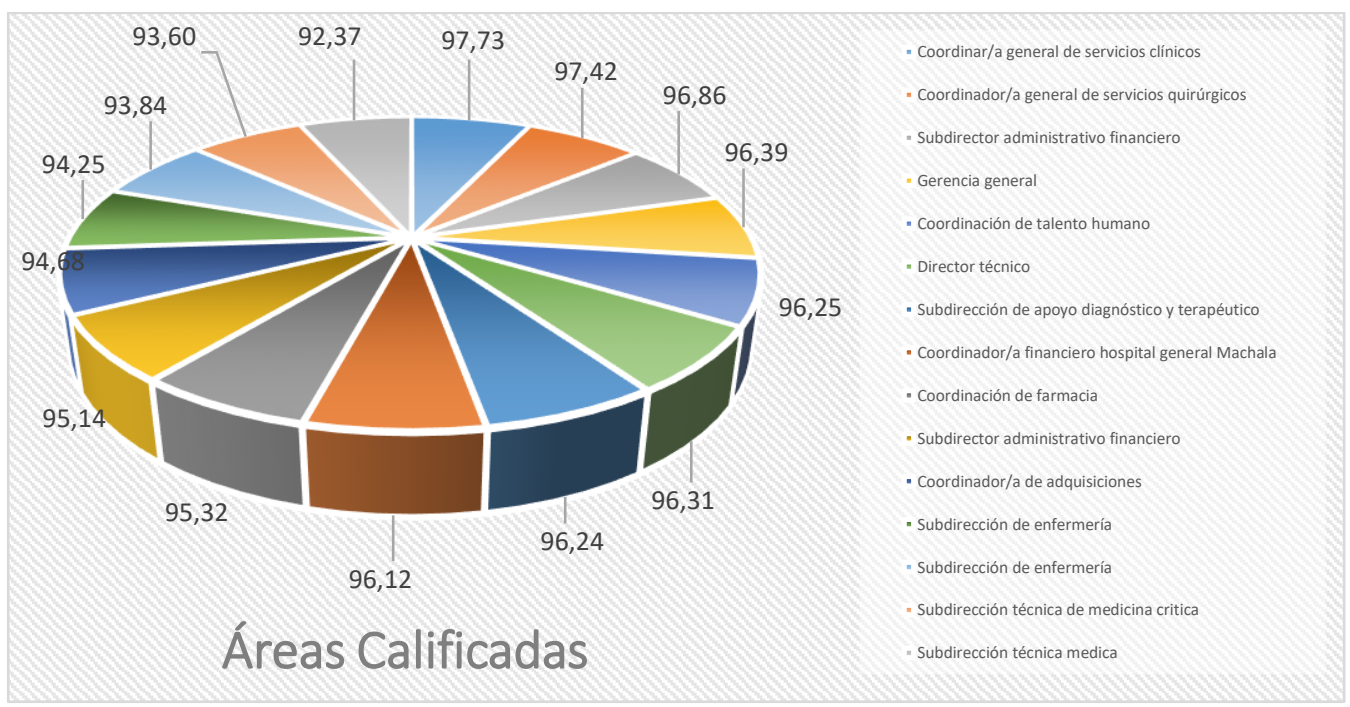

Figura 2. Porcentaje de áreas calificadas del HGM. 
CIENCIAMATRIA

Revista Interdisciplinaria de Humanidades, Educación, Ciencia y Tecnología

Año VII. Vol. VII. N¹2. Enero - Junio. 2021

Hecho el depósito de ley: pp201602FA4721

ISSN-L: 2542-3029; ISSN: 2610-802X

Universidad Nacional Experimental Francisco de Miranda (UNEFM). Santa Ana de Coro. Venezuela

Karen Lisseth Apolo-Pérez; Juan Edmundo Álvarez-Gavilanes;

William Giovanni Vicuña-Matute

Fuente: Investigación de campo.

La figura 2 representa las áreas con mayor porcentaje obtenido de la evaluación de desempeño, obteniendo como resultado a los tres mayores puntajes, según se detalla:
1. Coordinar/a general de servicios clínicos
$97,73 \%$
2. Coordinador/a general de servicios quirúrgicos
$97,42 \%$
3. Subdirector administrativo financiero
$96,86 \%$

\section{CONCLUSIONES}

Una vez realizada la investigación, se concluye que el talento humano del HGM no ha sido capacitado en las áreas que se desempeña, lo que está generando que necesiten mayor tiempo para realizar los procesos, ocasionando que no se estén cumpliendo con los objetivos planteados.

Se define que existe un escenario ideal para realizar propuestas internas orientadas al mejoramiento continuo de la gestión del talento humano.

Con el objetivo de contribuir a la mejora de la formación del talento humano, se enfocó en capacitar al personal, además de evaluar y medir el desempeño de sus actividades dando como resultado contar con un personal formado y altamente capacitado en temas específicos de las actividades que realiza, lo que permitirá mejorar la productividad individual para la consecución de metas a nivel departamental.

Con la aplicación de esta propuesta mejorará el clima laboral actual donde además se le reconocerá su esfuerzo y compromiso lo que aportará al mejoramiento de los procesos como el logro de los objetivos institucionales que es mejorar el servicio para la satisfacción de los afiliados. 


\section{REFERENCIAS}

Alonso-Suárez, C., Parra-Rojas, R., \& Arronte-Cruz, L. (2010). Un sistema de gestión del capital humano en la EMCE basado en las competencias laborales [A human capital management system at the EMCE based on labor competencies]. Ingeniería Industrial, 31(1).

Alveiro-Montoya, C. (2009). Evaluación del desempeño como herramienta para el análisis del capital humano. Revista Científica "Visión de Futuro", 11(1).

Arellano - Narváez, M. S. (2016). Diseño del cuadro de mando operativo mediante la integración de los subsistemas de selección, capacitación y gestión del desempeño. Caso: la casita de chocolate de la ciudad de Quito, 2013. http://repositorio.puce.edu.ec/handle/22000/9589

Armijos-Mayon, F. B., Bermúdez-Burgos, A. I., \& Mora-Sánchez, N. V. (2019). Gestión de administración de los recursos humanos [Human resources management]. Universidad Y Sociedad, 11(4), 163-170.

Ascencio-Vargas, A., Campo-Ramírez, J., Ramírez-Roldán, A., \& Zapata-Cardona, L. (2016). Importancia de la planeación estratégica en las áreas de gestión humana de las organizaciones [Training in university management to achieve labor competencies in the administrative staff of the Universidad Nacional Autónoma Altoandina de Tarma, 2017]. Revista Fundación Universitaria Luis Amigó (histórico), 3(1), 116-122. https://doi.org/10.21501/23823410.1899

Bernal-González, I., Pedraza-Melo, N., \& Sánchez-Limón, M. (2014). El clima organizacional y su relación con la calidad de los servicios públicos de salud: Diseño de un modelo teórico [The organizational climate and its relationship with the quality of public health services: Design of a theoretical model]. Estudios Gerenciales, 31(134); 8-19. https://doi.org/10.1016/j.estger.2014.08.003

Calderón-Hernández, G., \& Naranjo-Valencia, J. (2004). Competencias laborales de los gerentes de talento humano. INNOVAR. Revista de Ciencias Administrativas y Sociales, (23),79-97.

Capuano, A. (2004). Evaluación de desempeño: desempeño por competencias. Invenio, $7(13), 139-150$. 
Chávez-Epiquén, A., Moscoso Paucarchuco, K. M. y Manrique Chávez, Z. R. (2018) «Capacitación en gestión universitaria para lograr competencias laborales en el personal administrativo de la Universidad Nacional Autónoma Altoandina de Tarma, 2017», Horizonte de la Ciencia, 8(14); 131-139.

Cordero, J., Narváez, C., \& Erazo, J. (2019). La evaluación por competencias: una herramienta para determinar la productividad del talento humano. 593 Digital Publisher CEIT, 4(3-1), 76-96.

Espín-Oleas, M. E., Zula-Cujano, J. A., \& Carrión-Erazo, L. E. (2015). Gestión del talento humano orientado al alto desempeño de los servidores públicos [Human talent management oriented to the high performance of public servants]. CCCSS Contribuciones a las Ciencias Sociales, 16. Recuperado de https://n9.cl/tbl38

Gonzáles-Ramos, M., \& Molina-Gómez, A. (2016). Gestión del talento humano: Reflexiones desde la atención primaria de salud [Human Talent Management: Reflections from Primary Health Care]. MediSur, 14(2), 97-100.

Gutiérrez-Alvarado, J. J. (2011). La gestión del talento y la generación de valor de la empresa [The management of the talent and the generation of value of the company.]. Recuperado https://repositorioacademico.upc.edu.pe/handle/10757/333455

Instituto Ecuatoriano de Seguridad Social. (2017). Resolución No. C.D. 546. Recuperado de https://n9.cl/m79ro

Jurado-de-los-Santos, P. (2006). Análisis de necesidades formativas de los profesionales de centros ocupacionales y centros especiales de trabajo en Cataluña [Analysis of the training needs of professionals in occupational centers and special work centers]. Educar, 38; 81-103.

López-Puig, P., Díaz-Bernal, Z., Segredo-Pérez, A. M., \& Pomares-Pérez, Y. (2017). Evaluación de la gestión del talento humano en entorno hospitalario cubano [Evaluation of human talent management in Cuban hospital environment]. Revista Cubana de Salud Pública, 43(1),3-15. 
Manjarrés, A., Castell , R., \& Luna, C. (2013). Modelo de evaluación del desempeño basado en competencias [Competency-based performance evaluation model]. Ingeniare, (15), 11-29. https://doi.org/10.18041/1909-2458/ingeniare.15.598

Matabajoy-Montilla, J. M., Matabachoy-Tulcán, S. M., \& Obando-Guerrero, L. M. (2017). Procesos de desarrollo del talento humano en una clínica de especialidades de Pasto, Colombia [Human talent development processes in a specialty clinic in Pasto, Colombia]. Universidad y Salud, 20(1), 26 - 36. https://doi.org/10.22267/rus.182001.106

Mejía-Giraldo, A., Bravo-Castillo, M., \& Montoya-Serrano, A. (2012). El factor del talento humano en las organizaciones [Human talent factor in the organizations]. Ingeniería Industrial, 34(1), 2-11.

Mendivel-Gerónimo, R., Lavado-Puente, C., \& Sánchez-Castro, A. (2020). Gestión administrativa y gestión de talento humano por competencias en la Universidad peruana Los Andes, Filial Chanchamayo [Administrative management and human talent management by competencies at the Peruvian University Los Andes, Chanchamayo Branch]. Revista Conrado, 16(72), 262-268.

Mendoza-Novillo, P. A., Erazo-Álvarez, J. C., \& Narváez-Zurita, C. I. (2019). Estudio de tiempos y movimientos de producción para Fratello Vegan Restaurant [Study of production times and movements for Fratello Vegan Restaurant]. CIENCIAMATRIA, 5(1), 271-297. https://doi.org/10.35381/cm.v5i1.267

Miranda-Hoyes, D. (2016). Motivación del talento humano: La clave del éxito de una empresa [Motivation of human talent: The key to the success of a company]. Revista Investigación y Negocios, 9(13), 20-27.

Montenegro, I. (2007). Evaluación del desempeño docente. Fundamentos, modelos e instrumentos [Teacher performance evaluation. Foundations, models and instruments]. Bogotá, Colombia: Cooperativa Editorial Magisterio. doi:https://n9.cl/sifnl

Pérez T., C, \& Vera Méndez, F. (2011). Indicadores de gestión enfocados al ahorro energético para la industria de beneficio de feldespato. Scientia Et Technica, $\mathrm{XVI}(49), 72-77$. 
Ramírez, R., Espindola , C., Ruíz, G., \& Hugueth, A. (2019). Gestión del Talento Humano: Análisis desde el Enfoque Estratégico [Human Talent Management: Analysis from the Strategic Approach]. Información tecnológica, 30(6), 167-176.

Rivero, A. G., \& Dabos, G. E. (2017). Gestión diferencial de recursos humanos: Una revisión e integración de la literatura [ Differential Human Resource Management: A Literature Review and Integration]. Estudios Gerenciales, 33(142), 3951. https://dx.doi.org/10.1016/j.estger.2016.12.003

Rivero-Ramírez, Y. (2019). Evaluación del desempeño: tendencias actuales [Performance evaluation: current trends]. Revista Archivo Médico de Camagüey, 23(2), 159-164.

Rodríguez, D., Erazo, J., \& Narváez, C. (2019). Técnicas cuantitativas de investigación de mercados aplicadas al consumo de carne en la generación millennial de la ciudad de Cuenca (Ecuador) [Quantitative market research techniques]. Revista Espacios, 40(32), 20.

Romero-Parra, L., \& Salcedo-Lara, M. (2019). Estrategias para fortalecer el proceso de gestión del talento humano en una entidad pública [Strategies to strengthen the human talent management process in a public entity]. SIGNOS - Investigación En Sistemas De gestión, 11(2), 99-117. https://doi.org/10.15332/24631140.5084

Ruiz de Vargas, M, \& Jaraba Barrios, B, \& Romero Santiago, L. (2005). Competencias laborales y la formación universitaria. Psicología desde el Caribe, (16),64-91.

Soto-Berenguel, A. (2003). El análisis de necesidades formativas en organizaciones a través del paradigma de la Ciencia Pragmática [The analysis of training needs in organizations through the paradigm of Pragmatic Science]. Revista de Psicología del Trabajo y de las Organizaciones, 19(3),277-292.

Valencia, D., Erazo, J., \& Narváez, C. (2019). El clima organizacional y su incidencia en la motivación del Talento Humano [The organizational climate and its impact on the motivation of human talent] Recuperado: https://n9.cl/ncuiz. Revista Arbitrada Interdisciplinaria Koinonía, 4(1), 436-467. http://dx.doi.org/10.35381/r.k.v4i1.464 


\section{CIENCIAMATRIA}

Revista Interdisciplinaria de Humanidades, Educación, Ciencia y Tecnología

Año VII. Vol. VII. N¹2. Enero - Junio. 2021

Hecho el depósito de ley: pp201602FA4721

ISSN-L: 2542-3029; ISSN: 2610-802X

Universidad Nacional Experimental Francisco de Miranda (UNEFM). Santa Ana de Coro. Venezuela

Karen Lisseth Apolo-Pérez; Juan Edmundo Álvarez-Gavilanes;

William Giovanni Vicuña-Matute

Vera-Barbosa, A., \& Blanco-Ariza, A. B. (2019). Modelo para la gestión del talento humano en las pymes del sector servicios de Barranquilla, Colombia [Model for human talent management in SMEs in the service sector in Barranquilla, Colombia]. Innovar, 29(74), 25-44.

C2021 por los autores. Este artículo es de acceso abierto y distribuido según los términos y condiciones de la licencia Creative Commons Atribución-NoComercial-Compartirlgual 4.0 Internacional (CC BY-NC-SA 4.0) (https://creativecommons.org/licenses/by-nc-sa/4.0/). 